(2) Open Access Full Text Article

\title{
Rewards, recognition, and caregiver engagement at Cleveland Clinic
}

\author{
Matthew E Majernik \\ Joseph M Patrnchak \\ Cleveland Clinic, Cleveland, OH, USA
}

Correspondence: Joseph M Patrnchak Cleveland Clinic, 9500 Euclid Avenue, NA \#4, Cleveland, OH 44195, USA

Tel +l 2164441917

Fax +I 2164440088

Email jp@jpatrnchak.com
This article was published in the following Dove Press journal:

Journal of Healthcare Leadership

22 April 2014

Number of times this article has been viewed

Abstract: Organizations with high levels of employee engagement have been shown to significantly outperform those with lower engagement. In health care organizations, the level of engagement affects such critical metrics as quality, safety, and patient satisfaction. Being recognized for good work has been identified as a key driver of employee engagement. With this in mind, Cleveland Clinic has established a "Total Rewards" strategy as a core component of its ongoing drive to increase employee engagement - an effort that has produced dramatic positive results since its launch in 2008. In addition to an overview of the clinic's Total Rewards strategy, this paper focuses in particular on "Caregiver Celebrations," the clinic's innovative employee recognition program. The success of Caregiver Celebrations can be measured by its high rates of utilization, by positive anecdotal feedback from both managers and caregivers, and by data from the clinic's annual engagement survey.

Keywords: health care, employee engagement, employee recognition

\section{Cleveland Clinic}

Perennially ranked among the top US hospitals, Cleveland Clinic, Cleveland, $\mathrm{OH}$, USA was ranked number four by U.S. News and World Report in 2013, with 15 of the clinic's specialties rated among the nation's best. ${ }^{1}$ US News has ranked the clinic number one in cardiac care for the past 19 years. ${ }^{1}$

Cleveland Clinic includes the Cleveland main campus; eight regional hospitals in Northeast Ohio; 16 family health and ambulatory surgery centers; and facilities in Florida and Nevada, Canada, and Abu Dhabi. In 2012, admissions across the system exceeded 160,000 .

Today, Cleveland Clinic has over 44,000 employees, including 3,100 physicians and scientists and 11,000 nurses. The clinic is distinctive for its staff model, in which all physicians are on salary, and for its delivery model, in which related medical specialties are grouped into 27 unified institutes offering patient-centered care. Renowned for its numerous medical innovations, the clinic attracts patients from all 50 US states and from over 130 countries.

But in 2008, despite its international prominence and undisputed clinical excellence, the clinic had to face the fact that its performance was decidedly below average in two areas. In March of that year, the results of the first federal Hospital Consumer Assessment of Healthcare Providers and Systems (HCAHPS) survey were announced and the clinic scored just at or below the national average in most of the categories of patient satisfaction (although the clinic did score slightly higher than average in its overall rating and in the "would recommend" category). ${ }^{2}$ Then, just a few months later, the results of 
a Gallup, Inc. (Washington, DC, USA) survey of employee engagement at the clinic ranked the clinic only in the 44th percentile of health care systems in Gallup's global database (Gallup/Cleveland Clinic, unpublished data 2008).

In short, in 2008, neither patients nor employees were fully satisfied with their experience at Cleveland Clinic. To the clinic's leaders, that was unacceptable, and major initiatives were launched to improve both employee engagement and patient satisfaction.

\section{Employee engagement}

Employee engagement has been defined as:

[...] a heightened emotional and intellectual connection that an employee has for his/her job, organization, manager, or co-workers that, in turn, influences him/her to apply additional discretionary effort to his/her work. ${ }^{3}$

As to the effect of that "heightened emotional and intellectual connection" on organizational performance, a Gallup meta-analysis of 199 research studies found that higher levels of engagement correlate with such metrics as customer loyalty, profitability, productivity, turnover, safety incidents, shrinkage, absenteeism, and quality. ${ }^{4}$ Specifically with respect to health care institutions, the same analysis found that units in the upper quartile in engagement had a median $41 \%$ fewer patient safety incidents than units in the bottom quartile. ${ }^{4}$ In terms of engagement effects on customer-ie, patient - satisfaction, another Gallup study examined 94 hospitals with more than 130,000 employees and found that "hospitals with higher levels of engagement also register higher HCAHPS domain performance." 5

The question, of course, is why some employees are more engaged than others, and why some organizations have more engaged workforces than others. In other words, what drives employee engagement? Gallup, which has arguably devoted more attention than any other institution to this question, has identified 12 key drivers. Integrated into Gallup's proprietary $\mathrm{Q}^{12 ®}$ engagement survey instrument, these include whether an employee's supervisor "seems to care about me as a person," whether or not the employee has received recognition in the recent past, and whether the organization's mission "makes me feel my job is important." Other issues covered by the $\mathrm{Q}^{12}$ include the employee's knowing what is expected of him/her, feeling as if "my opinions seem to count," having the materials needed to succeed, and being encouraged (and having the opportunity) to develop professionally. ${ }^{4}$

\section{Employee engagement at Cleveland Clinic}

Recognizing the connection between employee engagement and patient satisfaction, and using Gallup's $\mathrm{Q}^{12}$ framework as a guide, Cleveland Clinic in 2008 committed itself to making the clinic a "great place to work and grow." This effort has evolved to include a number of key components, including the rewards and recognition strategy that is the focus of this paper. Before moving on to rewards and recognition, the following provides a brief outline of the clinic's other engagement initiatives. A more comprehensive discussion is available in "Building an engaged workforce at Cleveland Clinic," a journal article by one of the co-authors of this paper. ${ }^{6}$

\section{"We are all caregivers"}

The term "caregiver" has traditionally referred to individuals who provide direct care to a patient, whether physicians and nurses, home health care aides, or even family members. Recognizing, however, that a patient's hospital experience includes more than just his/her clinical outcome, and that, therefore, every hospital employee can affect the quality of that overall experience, one of Cleveland Clinic's first engagement initiatives was to embrace the concept of "we are all caregivers." "Employee" has been changed to "caregiver" on all communications materials, from ID badges to the clinic website, and the "we are all caregivers" theme is constantly highlighted in print and electronic media throughout the clinic. To reinforce a caregiver mindset, the clinic developed a "Cleveland Clinic Experience" program, in which every caregiver has participated. Meeting for a half-day session, groups of eight to ten caregivers from different departments and levels - physicians, nurses, food service workers, executives, managers, entry level staff - focus on how to respond with greater empathy to both colleagues and patients. To sustain the lessons learned, follow-up activities at the unit level are conducted on a regular basis.

\section{Serving leadership}

To ensure that managers' behavior actually reflects the "we are all caregivers" concept, the clinic has made a concerted effort to complement what has been predominantly a command and control leadership model with serving leadership principles. All 3,100+ leaders in the organization have received serving leader training focused on the leader's responsibility to help others succeed and realize their full potential. ${ }^{7,8}$ Serving leader behaviors have been included in the clinic's performance management tool. Some 120+ serving leader champions have been extensively trained to serve 
as mentors and support the use of serving leader principles in their institutes/divisions/departments.

\section{Wellness and well-being: caring for the caregivers}

To reinforce the message that the clinic does indeed "care for the caregiver" - and not coincidentally to also reduce the organization's employee health care costs - the clinic has developed a proactive caregiver wellness strategy. In 2008, the clinic began offering caregivers free access to weight-loss/ fitness programs provided by Curves (Woodway, TX, USA) and Weight Watchers (New York, NY, USA) with a \$100 cash incentive for participation. The clinic has since restructured its employee health plan so that caregivers who participate and achieve goals in the wellness programs can save up to $13 \%$ on their health plan premiums.

To date, more than $50 \%$ of the clinic's caregivers have participated in its wellness programs. Participation in the clinic's chronic disease management programs targeting obesity, asthma, diabetes, hypertension, and hyperlipidemia has increased by $825 \%$, to four times the national average. In addition to the engagement effect and excellent clinical results, measured by such factors as reduced hospital admissions, the wellness initiative has produced savings/cost avoidance of US\$78 million since 2010 (Cleveland Clinic, unpublished data, 2013).

\section{Rewards and recognition at Cleveland Clinic}

Another key component of Cleveland Clinic's ongoing engagement initiative is a "Total Rewards" strategy that integrates an array of monetary/nonmonetary tools. In addition to the "Caregiver Celebrations" program, which is the primary focus of this paper, these include comprehensive health coverage (health, prescription drug, dental, vision), retiree medical plan, pension/investment programs, generous tuition reimbursement, and a variety of other programs such as adoption assistance, emergency financial support, home purchase assistance, and computer purchasing/ employee discount programs. Despite significant financial constraints, compensation for a number of previously undercompensated positions in nursing and allied health (eg, pharmacy, radiology, physical therapy) has been raised to market level.

Every clinic caregiver has access to "My Total Rewards," an up-to-date online summary of his/her own Total Rewards package, including the current cash value of base pay, benefits, retirement, wellness programs, and awards.

\section{Recognition drives engagement}

Recognition at work is a significant factor in driving engagement, as indicated by Gallup's including "recognition in the past seven days" as one of its $\mathrm{Q}^{12}$ engagement metrics. A Watson Wyatt (now Towers Watson, New York, NY, USA) study of engagement declared that "[...] manager-delivered recognition of employee performance boosts engagement the way a turbocharger cranks up a sports car's horsepower."9 This same study reported that, in organizations with low levels of engagement, "[...] strong manager performance in recognizing employee performance increases engagement by almost 60\%.",

Another Watson Wyatt survey noted that "the increased use of recognition programs is one way to help keep key talent engaged and motivated". ${ }^{10}$ Derek Irvine argues that recognition programs can "empower companies to create a unified, global workforce". ${ }^{11}$ In their book on the subject of strategic recognition, Mosley and Irvine maintain that:

Done right, recognition communicates the right behaviors [...] reinforces those behaviors, and gives a sense that the organization values employee contributions. Done right, recognition is management's positive way of creating engagement. ${ }^{12}$

In a similar vein, Molinaro and Weiss, commenting on their research into the drivers of employee engagement, note that:

Recognition is another important driver of employee engagement. Recognition may mean monetary rewards and compensation, but it also can refer to the appreciation and direct feedback that employees receive from managers. This recognition and appreciation demonstrates that employees are valued. ${ }^{13}$

\section{Caregiver Celebrations}

Prior to 2010, Cleveland Clinic had no consistent, enterprisewide recognition program. Then, in June of that year, the clinic launched Caregiver Celebrations. Mosley and Irvine, in the previously cited book on strategic recognition, stress that the most effective corporate recognition programs are tied to the organization's core values. ${ }^{12}$ Caregiver Celebrations was explicitly designed to recognize outstanding behaviors and performance that support Cleveland Clinic's values:

- Quality: maintains the highest standards and achieves them by continually measuring and improving outcomes.

- Innovation: welcomes change, encourages invention, and continually seeks better, more efficient ways to achieve goals. 
- Teamwork: collaborates and shares knowledge to benefit patients and/or fellow caregivers for the advancement of our mission.

- Service: strives to exceed patients' and/or fellow caregivers' expectations.

- Integrity: adheres to high moral principles and professional standards by a commitment to honesty, confidentiality, trust, respect, and transparency.

- Compassion: demonstrates a commitment to world-class care by providing a caring, supportive environment for patients, patients' families, and fellow caregivers (Cleveland Clinic, data on file, 2010).

Caregiver Celebrations is structured into several award tiers:

1. Appreciation: nonmonetary commendation from a manager, peer, or patient.

2. Honors: cash award from $\$ 10$ to $\$ 100$; manager-driven. Managers have an annual award budget of $\$ 25$ per direct report.

3. Excellence: $\$ 250$ cash or gift certificate, plus a desktop award, to $1 \%$ of the caregiver population each quarter; recipients nominated by institutes/divisions.

4. Caregiver: annually, 200 nominations enterprise-wide, with 50 awards of $\$ 2,000$ plus a desktop award.

5. CEO: annually, one individual award of $\$ 10,000$, one team award of \$10,000 (Cleveland Clinic, data on file, 2013).

Recipients of the Caregiver and CEO awards are also celebrated by the CEO and other top leaders at the clinic's annual Caregiver Celebrations banquet.

Designers of the program have made every effort to make the recognition process quick and easy. Caregivers, managers, and patients can use a simple Caregiver Celebrations website to submit recognitions and award nominations. Patients can also fill out a printed patient comment card and drop it into one of the many boxes around the clinic designated for that purpose. Senior leaders can also fill out an "Appreciation Award" certificate on the spot as they visit units across the clinic as part of the monthly "leadership rounding" program.

Over time, the Caregiver Celebrations website has been enhanced to include features such as a "Raves and Recognition Wall' that lists each institute's/division's 50 most recent awards, and a manager page that alerts managers to upcoming birthdays and service anniversaries in their units.

Considerable effort has gone into branding and publicizing the program. The clinic's Total Rewards team encourages managers to make Caregiver Celebrations a part of their dayto-day activities, and stories about how the program is being used across the organization are published in the quarterly Caregiver Celebrations newsletter. Award recipients are also highlighted in the newsletter, on video screens mounted throughout the clinic's many buildings, and at various events, including the highly publicized annual banquet where the CEO awards are presented.

Feedback on the program from managers has been very positive. As one manager noted: "Caregiver Celebrations gives leaders a consistent, reliable way to recognize employees. Because the program is easy to use, I find myself recognizing employees more often." Another commented that "Sending an award provides managers with an opportunity to engage, thank and recognize their employees and others, which is important in retaining staff." In a similar vein:

[...] this program is absolutely fantastic! It allows us to team build within our hospital [...] and it has been incorporated into our performance evaluations under "recognizing others for work well done and supports others to be their best."

The program's simplicity has produced high levels of utilization. In 2010, an average of 7,515 Caregiver Celebrations awards were delivered per month. Since then, the average number of awards per month has increased every year, to 8,864 (2011), 13,723 (2012), and 19,285 (2013). Since the program's launch in June 2010 to the end of November 2013, more than 535,000 awards were generated, comprising 376,000+ tier 1 Appreciation awards; 121,500+ tier 2 Honors awards; 2,700 tier 3 Excellence awards; 160+ tier 4 Caregiver awards; 16,300+ patient recognition awards; and six CEO awards (Cleveland Clinic, data on file, 2013).

Further evidence that Caregiver Celebrations has achieved its goal of providing the clinic with a consistent recognition framework is the fact that programs focused on such diverse issues as the patient experience, caregiver communications, caregiver wellness, and even telephone quality assurance are using Caregiver Celebrations to recognize those who help them achieve their objectives.

Anecdotal feedback indicates that the program has been enthusiastically received by caregivers and that it is having the desired effect on the perception of the clinic as a great place to work and grow. "It's very rewarding to know you are appreciated," wrote a clinical analyst in the Department of Nursing Informatics, who has received awards at the tier 1 and 2 levels, "many times words are spoken, but the award certificate is a nice addition to the spoken words." A nurse who received a tier 3 Excellence award commented: "The program reaffirms and validates what you're doing. It just makes you feel good." The fact that many award recipients 
display their awards on the walls of their workspace also indicates that the awards are a source of pride.

Some of the caregiver feedback suggests that in addition to its "feel-good" effect, Caregiver Celebrations also reinforces the "we are all caregivers" concept. For example, a financial analyst who received a tier 2 award from a Nursing Institute leader for her support in providing administrative data commented: "The award [...] helped me realize that even though the finance group has no direct patient contact, information we provide can still affect the experience and outcomes of our patients." Similarly, a member of the clinic's media group who received a Caregiver Celebrations award indicated that:

Even though we are in a nonclinical field, we strongly believe we are tied to the Cleveland Clinic experience, and receiving the award was a powerful confirmation of our role in putting patients and caregivers first.

A different perspective on the Caregiver Celebrations program emerged from a 2012 assessment of the clinic's Total Rewards strategy conducted by Aon Hewitt (Lincolnshire, IL, USA). Not surprisingly, considering that some $74 \%$ of Caregiver Celebration awards have no monetary component and another $23 \%$ have a value of only $\$ 10$ to $\$ 100$, Aon Hewitt found that, in terms of relative importance to caregivers, Caregiver Celebrations ranked below increased compensation and other Total Rewards components. On the other hand, Aon Hewitt also noted that Caregiver Celebrations - with its annual US\$2.5 million price tag, far less than other benefits - is a very cost-effective tool for improving caregiver percep- tions of the clinic. Thus, in developing its recommendations, the firm suggested that the clinic leverage "[the] low cost, high impact [Total Rewards] components, noting that for a modest cost, changes in dental, caregiver recognition, and LTD tend to produce better preference increases per dollar of cost" (Aon Hewitt/Cleveland Clinic, unpublished data, 2012). In short, Caregiver Celebrations delivers considerable "bang for the buck."

\section{Caregiver Celebrations drives Cleveland Clinic engagement}

While caregiver and manager feedback and Aon Hewitt's cost-benefit analysis provide useful insight into the value of the Caregiver Celebrations program, the most critical consideration must be its success in terms of engagement. In this regard, the results seem quite clear.

As indicated previously, one of the items on Gallup's $\mathrm{Q}^{12}$ engagement survey asks employees to respond to the statement "In the last seven days, I have received recognition or praise for doing good work." ${ }^{5}$ As shown in Figure 1, in Gallup's first engagement survey at the clinic, the score on this item was 3.24, based on a 5-point scale, with 1 being "strongly disagree" and 5 "strongly agree." This was the lowest score of the 12 survey items. In the second survey, conducted in 2009, the score on this item rose only to 3.26. Then, in June 2010, the clinic launched the Caregiver Celebrations program, and the Gallup score for that year on this item increased significantly, to 3.47. In 2011, the first full year of Caregiver Celebrations, the year-end survey indicated another statistically significant increase for this item, to 3.67.

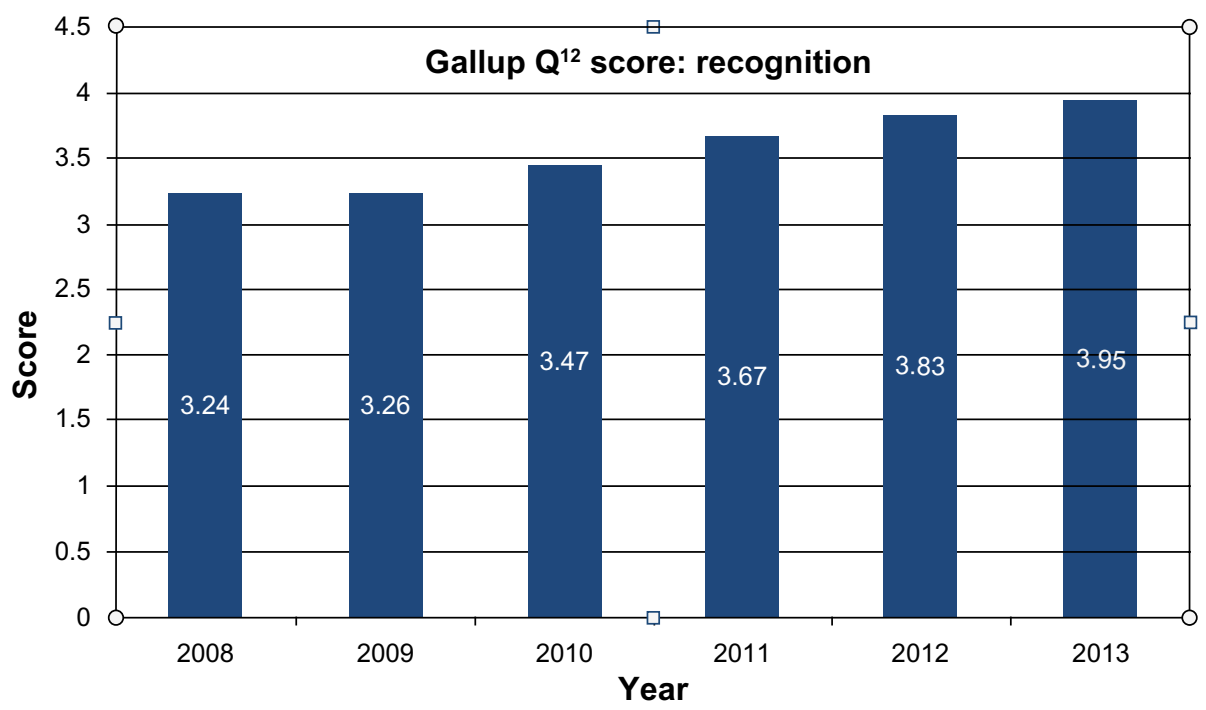

Figure I Employee engagement at Cleveland Clinic (Cleveland, OH, USA): $\mathrm{Q}^{12}$ score for "In the last seven days, I have received recognition or praise for doing good work." (Cleveland Clinic, data on file, 2013).

Note: $\mathrm{Q}^{12}$ : Gallup, Inc., Washington, DC, USA. 
In 2012, the score rose to 3.83, and to 3.95 for 2013. In each of those three years, the increase in the "recognition score" was the largest of any of the $\mathrm{Q}^{12}$ metrics. And overall, since the launch of Caregiver Celebrations in 2010, the clinic's score on the recognition item has increased more than any other $\mathrm{Q}^{12}$ item (Cleveland Clinic, unpublished data, 2013).

While causality cannot be proved, these results leave little room for doubt that Caregiver Celebrations has been responsible for the clinic's employees feeling that they receive more recognition for their work today than they did in the past. The very substantial increase in the clinic's score on the $\mathrm{Q}^{12}$ recognition metric also suggests strongly that the program and its effect on caregivers' perceptions of the clinic has had a significant positive impact on caregiver engagement.

The clinic's overall improvement in terms of caregiver engagement has been discussed in some detail in the previously cited "Building an engaged workforce at Cleveland Clinic," but the following key points should be made here.

As shown in Figure 2, Cleveland Clinic's overall score on the Gallup $\mathrm{Q}^{12}$ survey has increased from a grand mean of 3.80 in 2008 to 4.25 in 2013, a 5-year increase that Gallup characterizes as "best practice" level. When compared to the other hospital systems in the Gallup database, the clinic has risen from the 43rd percentile to the 87 th.

The Gallup survey also indicates that the ratio of engaged/ actively disengaged employees at the Clinic has shifted dramatically in a positive direction. According to Gallup, a 4:1 ratio is considered necessary for engaged employees to neutralize the negative effect of the actively disengaged, and "in world-class organizations the ratio of engaged to actively disengaged employees is 9.57:1." ${ }^{14}$ As shown in Figure 3, at Cleveland Clinic, the engaged/actively disengaged ratio has improved from 2.77:1 in 2008 to 10.2:1 in 2013.

Finally, it is worth noting that the improvement in the clinic's engagement level has been mirrored by a corresponding improvement in patient satisfaction. Figure 4 illustrates this correlation by mapping the clinic's engagement level compared to other hospital systems in the Gallup database against the clinic's overall HCAHPS rating in comparison to other hospitals in the HCAHPS database.

\section{Discussion}

Like other organizations, Cleveland Clinic has committed itself to building a more highly engaged workforce as a means of improving its organizational performance. Recognition for work well done is often cited as one of the key drivers of employee engagement, but making recognition an effective management tool in large organizations like Cleveland Clinic can be challenging. As Mosley and Irvine note: "For a large organization to implement an individual-recognition program in a sustainable way, it can't just depend on the enlightenment of every manager [...]." ${ }^{12}$ To meet this challenge and create an effective, sustainable recognition program, Cleveland Clinic has created Caregiver Celebrations.

Launched in 2010, this enterprise-wide recognition program targets behaviors that support the clinic's core values; it is designed for flexibility and ease of use, allowing peers, managers, and patients to recognize those behaviors via five levels of awards, both nonmonetary and monetary. The program and the award recipients themselves are highly publicized. Participation rates are high, and response to the program from both caregivers and managers has been enthusiastic. It seems fair to say that Caregiver Celebrations

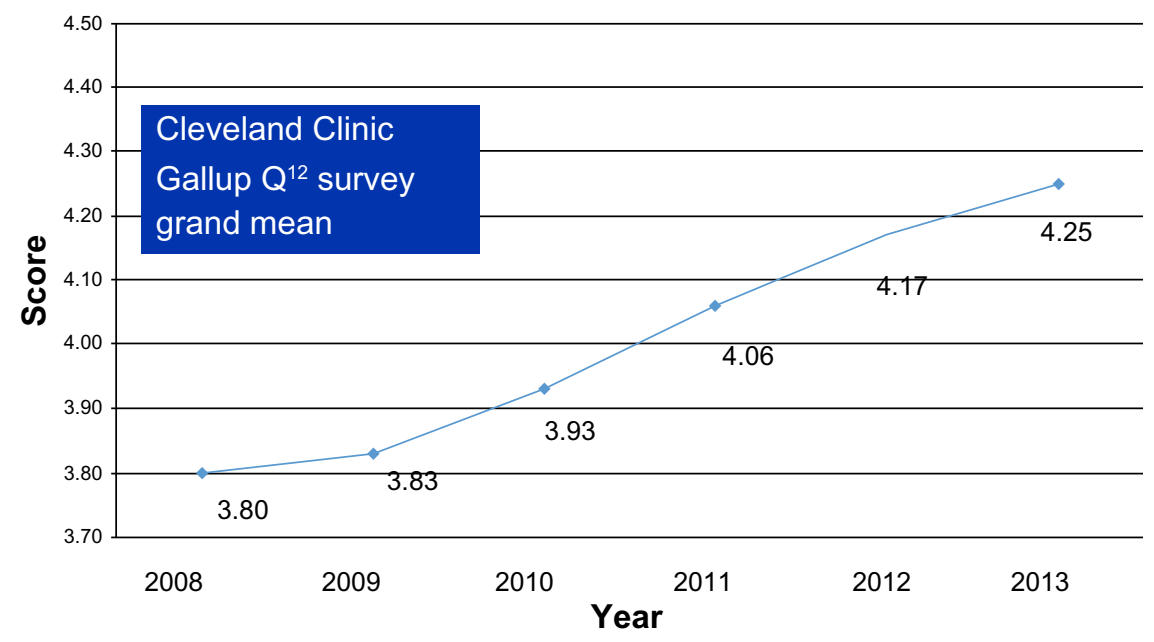

Figure 2 Employee engagement at Cleveland Clinic (Cleveland, OH, USA): overall $\mathrm{Q}^{12}$ score. Note: $\mathrm{Q}^{12}$ : Gallup, Inc., Washington, DC, USA. 
Ratio of engaged to actively disengaged caregivers

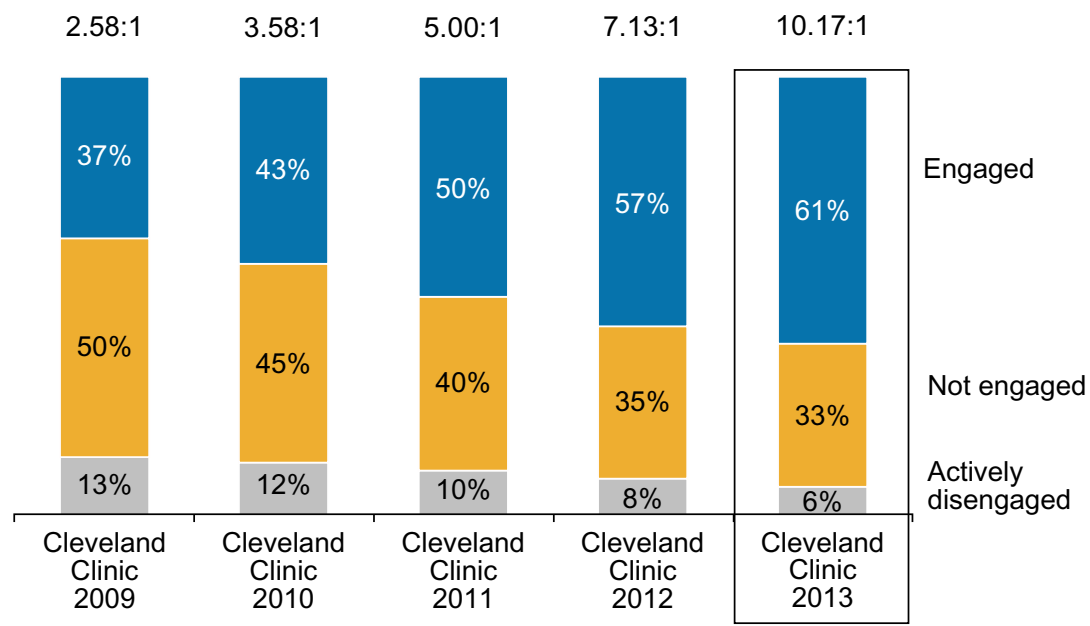

Figure 3 Employee engagement at Cleveland Clinic (Cleveland, OH, USA): engaged versus disengaged.

has become firmly embedded in the culture and day-to-day life of Cleveland Clinic. Most importantly, the program has been shown to have a significant positive impact on the clinic's employee engagement, which has increased dramatically in recent years.

A discussion of any large-scale employee recognition program should at least consider the possibility that such a program can backfire. In a recent Harvard Business School working paper, for example, the authors warn that in developing incentive/reward programs, it should be noted that "Perceptions of unfairness are known to motivate decreased effort and productivity." 15 They also raise the possibility that, in some cases, extrinsic rewards may "crowd out" intrinsic motivation and thus ultimately reduce performance and productivity. ${ }^{15}$

The fact that peer and patient recognition are included in Cleveland Clinic's Caregiver Celebrations program seems to mitigate against the perception of unfairness - specifically, the perception that managers favor certain employees in giving out awards. The large number of awards given out every year also seems to work against the perception that a "favored few" benefit inordinately from the program.

Similarly, the possibility that Caregiver Celebrations recognition might crowd out intrinsic motivation and ultimately reduce the desired behavior does not seem to be the case. One would reasonably expect that such an effect would

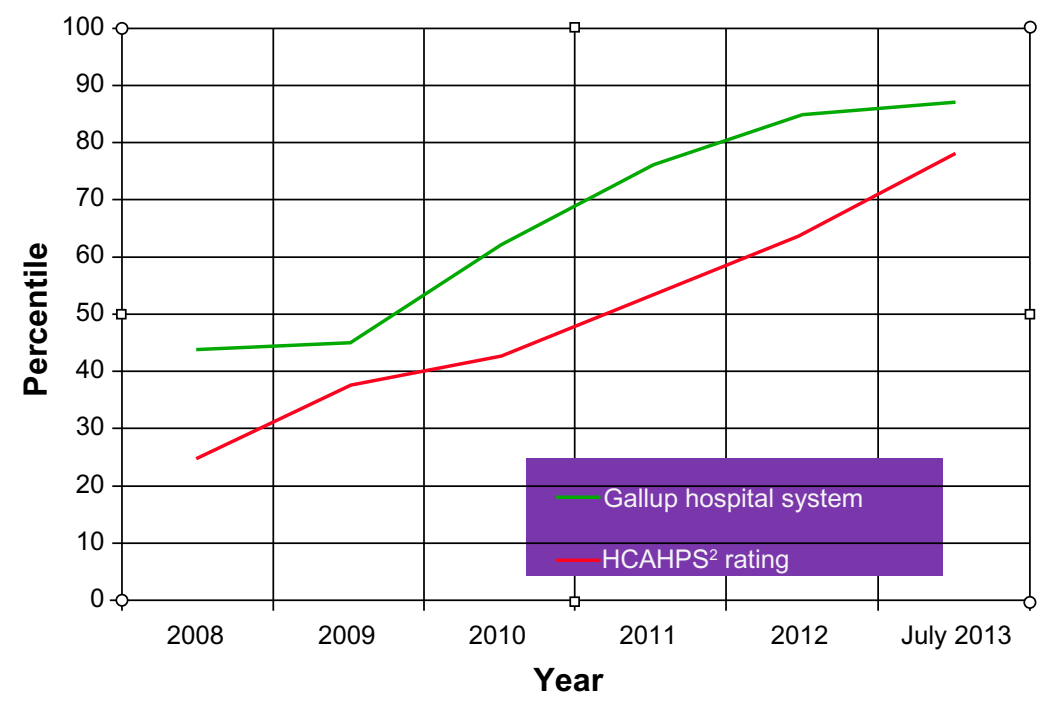

Figure 4 Employee engagement at Cleveland Clinic (Cleveland, OH, USA): engagement and patient satisfaction. Notes: Gallup, Inc., Washington, DC, USA

Abbreviation: HCAHPS, Hospital Consumer Assessment of Healthcare Providers and Systems. 
have been manifest in the 3 years since the introduction of Caregiver Celebrations. As already seen, however, the clinic's engagement levels since then have in fact increased substantially.

The lack of such a crowding out effect is also indicated by the clinic's $Q^{12}$ scores on "The mission or purpose of my organization makes me feel my job is important" item. In 2008 and 2009, the grand mean on this item remained unchanged, at 3.8. Since 2010, however, those scores have increased every year, to 4.31 in 2013. Other clinic engagement initiatives have undoubtedly contributed to this improvement, but Caregiver Celebrations certainly does not seem to have had a negative impact on this metric - a metric closely related to the intrinsic motivation of clinic employees.

Discussion of any initiative that purports to change organizational behavior must also consider the question of limitations and replicability: that is, what considerations might prevent these same results from being achieved in a different organizational setting. With initiatives that have been successful on a small scale, there is the question of scalability, but given the size of Cleveland Clinic, this seems to be a nonissue.

Cost is also a possible limitation. Clearly, there is some cost associated with Caregiver Celebrations, and this might be an issue for other organizations. As already noted, however, the program's cost is extremely modest as a percentage of the clinic's total benefits budget and minimal as a percentage of its total operating budget. It should also be noted that the vast majority of awards in the program have no or very little monetary component.

Perhaps the most important point to be made in terms of limitations is that Caregiver Celebrations is only one of several major engagement initiatives under way at Cleveland Clinic. It is not, and was not intended to be, a magic bullet that by itself would improve the organization's engagement levels. That said, given its relatively low cost and positive impact, a recognition program like Caregiver Celebrations seems to offer very substantial benefits to any large organization seeking to improve its performance which would seem to be virtually every such organization. The question almost seems to be not "why do it," but rather "why not do it?"

\section{Disclosure}

The authors report no conflicts of interest in this work.

\section{References}

1. US News Rankings 2012 [webpage on the Internet]. Cleveland, OH: Cleveland Clinic. Available from: http://my.clevelandclinic.org/p2/ us_news_rankings.aspx. Accessed March 20, 2013.

2. The Hospital Consumer Assessment of Healthcare Providers and Systems survey is the first national, standardized, publicly reported survey of patients' perspectives of hospital care. The HCAHPS survey asks discharged patients 27 questions about their recent hospital stay. For each participating hospital, ten HCAHPS measures are publicly reported. [webpage on the Internet.] Baltimore, MD: Centers for Medicare and Medicaid Services. Available from: http://www.hcahpsonline. org. Accessed June 6, 2013.

3. Gibbons J. Employee Engagement: A Review of Current Research and its Implications. New York, NY: The Conference Board, Inc.; 2006:5. Available from: http://montrealoffice.wikispaces.com/file/ view/Employee+Engagement+-+Conference+Board.pdf. Accessed February 20, 2012.

4. Harter JK, Schmidt FL, Killham EA, Agrawal S. Q12 ${ }^{\circledR}$ Meta-Analysis: The Relationship Between Engagement at Work and Organizational Outcomes. Washington, DC: Gallup, Inc.; 2009:26. Available from http://www.handsupincentives.com/wp-content/uploads/2011/10/ Employeeengagement_Q12_WhitePaper_2009.pdf. Accessed January 23, 2013.

5. Strengthening Care through Engagement. Washington, DC: Gallup, Inc.; 2009. Available from: http://www.gallup.com/ strategicconsulting/157250/strengthening-care-engagement.aspx. Accessed March 8, 2013.

6. Patrnchak JM. Building an engaged workforce at Cleveland Clinic. J Healthc Leadersh. 2013:5;9-20.

7. Greenleaf RK. The Servant as Leader. Westfield, IN: Greenleaf Center for Servant Leadership; 2008.

8. Jennings K, Stahl-Wert J. The Serving Leader: Five Powerful Actions that Will Transform Your Team, Your Business, and Your Community. San Francisco, CA: Berrett-Koehler Publishers, Inc.; 2003.

9. The Power of Recognition from Managers: Part 1: The Engagement Engine. Towers Watson; 2010; New York, NY. Available from: http:// www.towerswatson.com/en-US/Insights/IC-Types/Survey-ResearchResults/2009/12/Turbocharging-Employee-Engagement-The-Powerof-Recognition-From-Managers-Part-1. Accessed February 11, 2012.

10. Effect of the Economic Crisis on HR Programs. Watson Wyatt; 2009; Arlington, VA: 11. Available from: http://www.growbold.com/ files/wwhrprogramsaug09.pdf. Accessed February 11, 2012.

11. Irvine D. The Return on recognition in a recession. HR Management. February 16, 2012.

12. Mosley E, Irvine D. Winning with a Culture of Recognition. Southborough, MA: Globoforce Limited; 2010:34.

13. Molinaro, V, Weiss D. Driving Employee Engagement. Available from: http://www.banffcentre.ca/leadership/library/pdf/driving_employee_ engagement_Weiss-Molinaro.pdf. The Banff Centre. Accessed April 8, 2013.

14. How to create a culture of wellbeing. Gallup Business Journal. Washington, DC: Gallup, Inc.; 2013. Available from: http://business journal.gallup.com/content/159080/create-culture-organizationalwellbeing.aspx?ref=more. Accessed March 28, 2013.

15. Gubler T, Larkin I, Pierce L. The Dirty Laundry of Employee Award Programs: Evidence from the Field. Working Paper 13-069. Boston, MA: Harvard Business School; 2013. Available from: http://hbswk. hbs.edu/item/7215.html. Accessed December 8, 2013. 
Journal of Healthcare Leadership

\section{Publish your work in this journal}

The Journal of Healthcare Leadership is an international, peer-reviewed, open access journal focusing on leadership for the health profession. The journal is committed to the rapid publication of research focusing on but not limited to: Healthcare policy and law; Theoretical and practical aspects healthcare delivery; Interactions between healthcare and society and evidence-based practices; management; Research and opinion for health leadership; Leadership assessment. The manuscript management system is completely online and includes a very quick and fair peer-review system. Visit http://www.dovepress.com/ testimonials.php to read real quotes from published authors.

Submit your manuscript here: http://www.dovepress.com/journal-of-healthcare-leadership-journal 